\title{
Signal for a light singlet scalar at the LHC
}

\author{
We-Fu Chang, ${ }^{1,2}$ Tanmoy Modak, ${ }^{3}$ and John N. Ng${ }^{2}$ \\ ${ }^{1}$ Department of Physics, National Tsing Hua University, Hsinchu 30013, Taiwan \\ ${ }^{2}$ Theory Group, TRIUMF, Vancouver, British Columbia V6T2A3, Canada \\ ${ }^{3}$ Department of Physics, National Taiwan University, Taipei 10617, Taiwan
}

(Received 20 November 2017; published 15 March 2018)

\begin{abstract}
In the general Higgs portal-like models, the extra neutral scalar, $S$, can mix with the Standard Model (SM) Higgs boson, $H$. We perform an exploratory study focusing on the direct search for such a light singlet $S$ at the Large Hadron Collider (LHC). After careful study of the SM background, we find the process $p p \rightarrow t \bar{t} S$ followed by $S \rightarrow b \bar{b}$ can be used to investigate $S$ with mass in the $20<M_{S}<100 \mathrm{GeV}$ range, which has not been well explored at the LHC. The signal significance becomes meaningful with a luminosity around a few $\mathrm{ab}^{-1}$. Also, we study the prospects of finding the light scalar at the future $100 \mathrm{TeV} p p$ collider, the $Z$ and Higgs factories. With similar luminosity, the current Large Electron-Positron Collider (LEP) limits on the mixing between $S$ and $H$ can be improved by at least one or two order of magnitudes.
\end{abstract}

DOI: 10.1103/PhysRevD.97.055020

\section{INTRODUCTION}

The discovery of a $125 \mathrm{GeV}$ scalar with properties as expected of the Standard Model (SM) Higgs boson $H$ gives one last experimental support that it is the correct theory for physics at or below the Fermi scale. On the other hand, neutrino oscillations data points to massive active neutrinos which will require the model to be amended. Furthermore, the SM cannot account for the mounting evidence for dark matter and neither can it explain the matter-antimatter asymmetry of the Universe. Adding SM gauge singlet scalar fields is the simplest extension and the most economical and popular scenario to pursue. If one takes only one such complex field $S$ and minimally couples it to the SM via interactions with $H$, the beautiful successes of the SM in describing current data are largely undisturbed.

Theoretically, singlet scalar fields appear in many extensions of the SM. They form an essential part of the minimal Majoronic model that accommodates both dark radiation [1] and dark matter (DM) [2-4]. The singlet scalar is a good candidate for DM. In a different context, singlet scalar extension of the scalar sector helps make the electroweak phase transition more first order, which is crucial for a successful electroweak baryogenesis [5]. Moreover, the new bosonic degrees of freedom contribute to improving the vacuum stability of SM $[6,7]$.

Published by the American Physical Society under the terms of the Creative Commons Attribution 4.0 International license. Further distribution of this work must maintain attribution to the author(s) and the published article's title, journal citation, and DOI. Funded by SCOAP ${ }^{3}$.
In this paper, we take a simplified model point of view of the singlet Higgs model. By that, we assume there is only one experimentally accessible real SM singlet scalar which mixes with the SM Higgs. It may be part of a more complete theory, but we only focus on its property as a Higgs portal [8]. Its only interaction with the SM is via couplings to the Higgs field. It may have coupling to other hidden or dark sectors, see, e.g., [9]. The production cross section of such a light scalar at the collider is entirely determined by its mass and its couplings to the SM fields. Once the scalar is produced, the signal depends on its decay branching ratios. For the collider searches, the relevant parameters are the mass of the scalar, the mixing between the scalar and the SM Higgs, and its decay branching ratios. Details of how a particular model gives rise to the parameters mentioned above are unimportant for this study.

To set up our convention, we write the relevant Lagrangian adopted from [2,3] with a SM singlet scalar $S$ as

$$
\begin{aligned}
\mathcal{L} \supset & -\mu_{H}^{2}\left(H^{\dagger} H\right)+\lambda_{H}\left(H^{\dagger} H\right)^{2} \\
& -\mu_{S}^{2}|S|^{2}+\lambda_{S}|S|^{4}+\lambda_{S H}\left(H^{\dagger} H\right)|S|^{2} .
\end{aligned}
$$

Spontaneous symmetry breaking (SSB) takes place for both $H$ and $S$. Thus, $\langle S\rangle=v_{S} / \sqrt{2}$ and $\langle H\rangle=v_{H} / \sqrt{2}$ with $v_{H}=246 \mathrm{GeV}$. The mixing of $H$ and $S$ occurs. Writing $S=\left(v_{S}+s_{0}\right) / \sqrt{2}$ and $H=\left(0, v_{H}+h_{0}\right)^{T} / \sqrt{2}$, the mass square matrix in the basis $\left\{h_{0}, s_{0}\right\}$ reads

$$
\left(\begin{array}{cc}
2 \lambda_{H} v_{H}^{2} & \lambda_{H S} v_{H} v_{S} \\
\lambda_{H S} v_{H} v_{S} & 2 \lambda_{S} v_{S}^{2}
\end{array}\right)
$$


Denoting the mass eigenstates by $H_{m}, S_{m}$, we have

$$
\left(\begin{array}{c}
H_{m} \\
S_{m}
\end{array}\right)=\left(\begin{array}{cc}
c_{\theta} & -s_{\theta} \\
s_{\theta} & c_{\theta}
\end{array}\right)\left(\begin{array}{c}
h_{0} \\
s_{0}
\end{array}\right) .
$$

Here, we use the shorthand notation $s_{\theta}, c_{\theta}$ for $\sin \theta, \cos \theta$, and $\theta$ is the mixing angle. We would like to emphasize again that only $s_{\theta}$ is relevant for the direct collider searches. Our analysis does not depend on the origin of the mixings. In some other models, the mass square matrix needs not entirely come from SSB. The mixing satisfies

$$
\tan 2 \theta=\frac{\lambda_{S H} v_{H} v_{S}}{\lambda_{S} v_{S}^{2}-\lambda_{H} v_{H}^{2}},
$$

and we set the range of $\theta \in[-\pi / 2, \pi / 2]$. For light singlet, we are interested in the case that $\lambda_{S} v_{S}^{2}<\lambda_{H} v_{H}^{2}$, and the mass eigenvalues are

$$
\begin{gathered}
m_{H_{m}}^{2}=\lambda_{H} v_{H}^{2}+\lambda_{S} v_{S}^{2}+\sqrt{\left(\lambda_{H} v_{H}^{2}-\lambda_{S} v_{S}^{2}\right)^{2}+\lambda_{S H}^{2} v_{H}^{2} v_{S}^{2}}, \\
m_{S_{m}}^{2}=\lambda_{H} v_{H}^{2}+\lambda_{S} v_{S}^{2}-\sqrt{\left(\lambda_{H} v_{H}^{2}-\lambda_{S} v_{S}^{2}\right)^{2}+\lambda_{S H}^{2} v_{H}^{2} v_{S}^{2}} .
\end{gathered}
$$

The convention is set so that when $\lambda_{S H}=0$, i.e., no mixing between $H$ and $S$, the above go back to $m_{H_{u}}^{2}=2 \lambda_{H} v_{H}^{2}$ and $m_{S_{m}}^{2}=2 \lambda_{S} v_{S}^{2} . H_{m}$ is identified as the $125 \mathrm{GeV}$ SM-like Higgs boson, and $S_{m}$ is the new neutral scalar boson. $M_{H}$ is the mass of $H_{m}$ which is $125 \mathrm{GeV}$, whereas the mass $M_{S}$ of $S_{m}$ is unknown. For notational simplicity, we drop the subscript $m$ for the mass eigenstates.

The main goal of our work is to give a careful study of the constraint on $M_{S}$ and $s_{\theta}$ from LHC and future colliders. Before we embark on this, it is well known that if the Higgs boson mixes with another scalar, independent of the mass of the unknown particle, the strength of the Higgs boson to other SM particles will be multiplied by $c_{\theta}$ at the amplitude level. This applies to $S$ with $c_{\theta} \rightarrow s_{\theta}$. The characteristic feature $S$ shares with the SM Higgs is that their couplings to the massive SM fields are proportional to the masses of the SM particles. Hence, $t, b, W, Z$ will be good choices for signal detection. This further implies that all Higgs boson signals currently measured at the LHC will have reduced SM-like strength. The SM signal strength is parametrized by $\mu$, and it is unity for the SM. The latest LHC- 1 bound is $\mu=1.09 \pm 0.11$ [10] which amounts to $\sin ^{2} \theta<0.13$ at $2 \sigma .{ }^{1}$ At LHC14, $\sin ^{2} \theta$ can be bounded to be less than $2 \times 10^{-2}$ from signal strength [11]. On the other hand, the discovery of $S$ depends crucially on $M_{S}$. The obvious mass effect is kinematical, i.e., the heavier $S$ is the lower the production rate at a given energy. The second effect is in the signals for $S$ detection. To give an example, for $M_{S}=400 \mathrm{GeV}$, the dominant decay mode will be

\footnotetext{
${ }^{1}$ At $2 \sigma$ C. L., $0.87<\mu=c_{\theta}^{2}<1.31$ implies that $s_{\theta}^{2}<0.13$.
}

$S \rightarrow W W, Z Z, t \bar{t}$ if the coupling to the hidden sector is not too large. In contrast, for a $40 \mathrm{GeV} S, \bar{b} b$ will be its dominant decay. Since the final states signals are drastically different, one needs to enhance the signal by optimizing $W$, $Z$, or $t$-quarks detection for the heavy $S$ case. For the $40 \mathrm{GeV}$ $S$, a more efficient $b$ quark tagging will help greatly. Another issue is the SM background suppression. For the example given above, it is clear that heavy and light scalars will require very different strategies for suppressing the backgrounds.

At the LHC, the dominant production mechanism for the SM Higgs and the scalar $(S)$ that mixed with it is via gluon fusion (GF). The subdominant production processes are the vector boson fusion $(\mathrm{VBF})$, the vector boson associated productions, $p p \rightarrow W^{ \pm} H / W^{ \pm} S, Z H / Z S$ (denoted as $W H(W S)$ and $Z H(Z S)$, correspondingly), and the topquark pair associated production $p p \rightarrow \bar{t} \bar{t} H / t \bar{t} S$ (denoted as $t \bar{t} H / t \bar{t} S)$. The production cross sections of VBF, $W H / W S, Z H / Z S$, and $t \bar{t} S$ are around $10^{-1}-10^{-2}$ times that of the GF. For the SM Higgs, or the scalar $S$ if it is lighter than $160 \mathrm{GeV}, b \bar{b}$ is the dominant decay modes. However, the overwhelming QCD background, mainly from $g g \rightarrow b \bar{b}$, $g g \rightarrow t \bar{t}$, and single top production $(t \bar{b})$, make the discovery challenging in this decay mode. Furthermore, when the scalar $S$ is heavier than $100 \mathrm{GeV}$, we have the luxury of using the rather rare but relatively clean decay modes such as $S \rightarrow 2 \gamma$ or $S \rightarrow Z Z^{(*)} \rightarrow \ell \bar{\ell} \ell \bar{\ell}$ for detection. If the scalar $S$ is heavier than $160 \mathrm{GeV}$, the decays $W W^{(*)}, Z Z^{(*)}$ provide additional handles for probing. The limit on the mixing between $S$ and $H$ is a by-product of direct searching for the SM Higgs at the LHC using the aforementioned clean signals $[12,13]$. The current limit is rather weak, $s_{\theta}^{2}<0.25$ for $80<M_{S}<600 \mathrm{GeV}$ except that $s_{\theta}^{2}<0.16$ when $M_{S}$ is in the window of $100<M_{S}<150 \mathrm{GeV}$.

However, when $M_{S}=40 \mathrm{GeV}$, the 2-photon decay branching ratio drops to $\sim \mathcal{O}\left(10^{-4}\right)$, and the branching ratio for $S \rightarrow 4 \ell$ drops to $\sim \mathcal{O}\left(10^{-7}\right)$. The $b \bar{b}$ decay is the only visible handle left to probe a light $S$ at the LHC. Still, the QCD backgrounds are prohibitive for the GF and VBF processes to be utilized. Moreover, subdominant processes such as $Z S, W S, t \bar{b} S$ are not useful for discovery due to huge $t \bar{t}$, single top, $W^{ \pm}+$and $Z+$ heavy flavor jets backgrounds. In order to discover such a scalar $S$ at the LHC, we turn our attention to subdominant production process $t \bar{t} S$, followed by $S \rightarrow b \bar{b}$ resonance with at least one top decaying leptonically. The high $b$-jet multiplicity, i.e., $>3 b$-jets, suppresses the dominant $t \bar{t}$ and single top backgrounds to a sufficiently low level, making $t \bar{t} S$ the most promising channel for the discovery of a low mass $S$ at the LHC. Given the smallness of the $t \bar{t} S$ production cross section, a high luminosity is required. A ballpark estimation is helpful here. To reach the sensitivity of $s_{\theta}^{2} \lesssim \mathcal{O}(0.01)$ for the light $\mathrm{S}$, the required luminosity is around $100 \times\left(\sigma_{G F} / \sigma_{t \bar{t} S}\right) \times(B r(S \rightarrow 2 \gamma) /$ $B r(S \rightarrow b \bar{b})) \times 10 \mathrm{fb}^{-1} \sim 10^{3} \mathrm{fb}^{-1}$. Such a luminosity is feasible at the high luminosity run of the LHC. 
In this paper, we focus on the range $20 \mathrm{GeV}<M_{S}<100 \mathrm{GeV}$. The lower limit is given by the energy resolution of LHC. Furthermore, due to the proximity of many bottomonium resonances in this energy range, our background analysis will not be applicable and the task of identifying a scalar resonance in the $b \bar{b}$ mode is nearly impossible. The upper limit is set by our choice of using multiple $b$-quarks as our signal for $S$ detection. For $M_{S}>100 \mathrm{GeV}$, the current LHC Higgs direct search strategies are more powerful than the $t \bar{t} S$ signal. As the luminosity increased to $\mathcal{O}\left(10^{3}\right) \mathrm{fb}^{-1}$, the current limit on $s_{\theta}^{2}$ from a direct search is expected to get an order of magnitude improvement as well. Moreover, searches in this low mass range at the LHC have not been covered by the existing studies on the general Higgs portal models [12-22].

We study the SM background in detail using the current knowledge of $b$-jets identification as documented by both ATLAS and CMS Collaborations. Currently, we have very limited knowledge on $S$ in this mass range. The only experimental information we have is the direct search limit from LEPII [23]. Hence, it is worthwhile to do a careful analysis to see how high luminosity (HL) LHC can improve on this. We also extrapolate to a future $100 \mathrm{TeV}$ hadron collider. As a comparison, we also study how Higgs and Z factory options can shed light on this issue by focusing on a few very clean reactions.

We note that for $M_{S}<10 \mathrm{GeV}$, stringent limits on $\sin ^{2} \theta$ are given by rare $B$ and $K$ mesons decays [24]. If $M_{S}<2 \mathrm{GeV}, \sin ^{2} \theta<10^{-6}$ from $B^{+} \rightarrow K^{+}+$nothing. When $M_{S}<0.36 \mathrm{GeV}, \sin ^{2} \theta<10^{-8}$ from $K^{+} \rightarrow \pi^{+}+$nothing. Thus, our study fills the gap between this and the heavy Higgs searches at the LHC.

The paper is organized as follows. In Sec. II, we summarize some relevant details of the simplified singlet scalar model. Section III gives the phenomenology of the model. Details of the LHC study are given. The chosen signals at the $\mathrm{Z}$ and Higgs factory options at the future $e^{+} e^{-}$ colliders are calculated. The constraints from muon $g-2$ and $B_{S} \rightarrow \mu \bar{\mu}$ are discussed as well. A summary and conclusions are given in Sec. IV.

\section{MODEL}

The Lagrangian for the simplified scalar singlet field is given in Eq. (1). The vacuum positivity requires that $\lambda_{H}$, $\lambda_{S}>0$, and $\lambda_{S H}>-2 \sqrt{\lambda_{H} \lambda_{S}}$. After SSB, one has

$$
\begin{aligned}
& \mu_{H}^{2}=\lambda_{H} v_{H}^{2}+\frac{\lambda_{S H}}{2} v_{S}^{2}, \\
& \mu_{S}^{2}=\lambda_{S} v_{S}^{2}+\frac{\lambda_{S H}}{2} v_{H}^{2} .
\end{aligned}
$$

In terms of $M_{H}(=125 \mathrm{GeV}), M_{S}, v_{S}$, and mixing, the scalar quartic couplings can be expressed as

$$
\begin{aligned}
\lambda_{H} & =\frac{1}{2 v_{H}^{2}}\left(c_{\theta}^{2} M_{H}^{2}+s_{\theta}^{2} M_{S}^{2}\right), \\
\lambda_{S} & =\frac{1}{2 v_{S}^{2}}\left(s_{\theta}^{2} M_{H}^{2}+c_{\theta}^{2} M_{S}^{2}\right), \\
\lambda_{S H} & =-\frac{s_{\theta} c_{\theta}}{v_{S} v_{H}}\left(M_{H}^{2}-M_{S}^{2}\right) .
\end{aligned}
$$

Among the mass eigenstates, the triple scalar coupling vertices are

$$
\begin{aligned}
& H H H:-3 i M_{H}^{2}\left(\frac{c_{\theta}^{3}}{v_{H}}-\frac{s_{\theta}^{3}}{v_{S}}\right), \\
& H H S:-i s_{\theta} c_{\theta}\left(\frac{c_{\theta}}{v_{H}}+\frac{s_{\theta}}{v_{S}}\right)\left(2 M_{H}^{2}+M_{S}^{2}\right), \\
& S S H:-i s_{\theta} c_{\theta}\left(\frac{s_{\theta}}{v_{H}}-\frac{c_{\theta}}{v_{S}}\right)\left(2 M_{S}^{2}+M_{H}^{2}\right), \\
& S S S:-3 i M_{S}^{2}\left(\frac{s_{\theta}^{3}}{v_{H}}+\frac{c_{\theta}^{3}}{v_{S}}\right) .
\end{aligned}
$$

In general (as long as $s_{\theta} / v_{H} \neq c_{\theta} / v_{S}$ ), the $H \rightarrow 2 S$ decay mode opens up when $M_{S}<M_{H} / 2$. Some weak constraints on $s_{\theta}$ can be derived from the SM Higgs total decay width, see for example, $[12,13]$. However, at the LHC, we do not consider the $p p \rightarrow H \rightarrow 2 S$ signal due to the smallness of the production cross section and the colossal SM background.

Since the quartic scalar couplings are irrelevant for our study, and they can be easily read from the Lagrangian, we do not spell them out here. The modifications to all the other SM Higgs-like couplings are straightforward: each $H / S$ will contribute one extra power of $c_{\theta} / s_{\theta}$ suppression factor. Note that the mixing is only between the two scalar parts; the Feynman rules for the Goldstone bosons remain unchanged.

When $M_{S}>2 m_{b}$, the dominating visible decay is $S \rightarrow b \bar{b}$. The partial width is given by

$$
\Gamma_{S \rightarrow b \bar{b}}=\frac{s_{\theta}^{2} N_{c} M_{S}}{8 \pi}\left(\frac{m_{b}}{v_{H}}\right)^{2}\left(1-4 \frac{m_{b}^{2}}{M_{S}^{2}}\right)^{3 / 2} .
$$

Taking into account the Large Electron-Positron Collider (LEP) limit on $s_{\theta}$ [23], the partial width is small, $\lesssim 10^{-4} \mathrm{GeV}$ for $M_{S}<80 \mathrm{GeV}$, and makes $S$ a very narrow resonance for discovery. There can be additional decays into invisible modes if $\mathrm{S}$ has a coupling to the dark sector. This will be model dependent.

\section{PHENOMENOLOGY}

\section{A. LHC signal}

In this subsection, we investigate the discovery potential of light $S$ at the LHC via $p p$ collision. The primary 
production mechanism of $S$ at LHC is via $p p \rightarrow t \bar{t} S$, followed by $S \rightarrow b \bar{b}$ decay and leptonic decays of at least one of the top quarks, constituting four $b$-tagged jets and at least one charged lepton, plus missing $\left(E_{T}^{\text {miss }}\right)$ signature. The final state topology is similar to SM $t \bar{t} H$ (with $H \rightarrow b \bar{b}$ ) production [25], which we closely follow in our analysis. The signal region will have subdominant contributions from single top productions such as $p p \rightarrow t S \bar{b} / j$ (conjugate processes implied). We also include several other processes, namely, $\quad p p \rightarrow \bar{t} W^{+} S, \quad p p \rightarrow W^{+} S H, \quad p p \rightarrow W^{+} S b \bar{b}$, $p p \rightarrow W^{+} Z S, p p \rightarrow Z Z S$, etc., which are possible within the model and provide small contributions to the signal region. There exists several sources of SM backgrounds, such as $t \bar{t}+$ heavy flavor jets ( $t \bar{t}+$ h.f. jets), $t \bar{t}+$ light flavor jets $(t \bar{t}+$ l.f. jets $)$ with subdominant contributions from $t$ - and $s$-channel single top production with contributions from $t \bar{t} Z, t \bar{t} H, t W H$, and other processes such as $Z / \gamma^{*}+4 b$-jets. We have not included nonprompt and fake backgrounds in our analysis. These contributions are not properly modeled in Monte Carlo event generators, and one needs data to estimate such contributions.

The signal and background samples are generated at leading order (LO) in Monte Carlo event generator MadGraph5_aMC@NLO [26] adopting NN23LO1 PDF set [27], interfaced with Pythia 6.4 [28] for showering, hadronization, and underlying events. The matrix element (ME) of the signal and background processes are generated up to one additional jet in the final state except for $t \bar{t}+$ heavy flavor jets. The ME for the latter is generated up to two additional jets in the final state. We follow MLM [29] matching prescription for the ME and parton shower (PS) merging. Due to computational limitations, we restrict the number of additional jets in ME to the above-mentioned numbers for the respective signal and background processes. Events are finally fed into Delphes 3.3.3 [30] for fast detector simulation (ATLAS based). The rejection factor for light jets and charm jets are assumed to be $1 / 137$ and $1 / 5$, respectively [31]. The jets are reconstructed using an anti- $k_{t}$ jet algorithm with radius parameter $R=0.5$. The effective model is implemented using FeynRules 2.0 [32].

Next, we discuss the selection cuts. Events are selected such that it should contain at least four jets out of which at least four are $b$-tagged and at least one is a charged lepton $(\equiv e, \mu)$ and missing transverse energy $E_{T}^{\text {miss }}$ (denoted as $(4 j, 4 b, 1 \ell)$ process). The $p_{T}$ of the leading lepton in an event is required to be $>25 \mathrm{GeV}$, while if it contains a subleading lepton, the transverse momenta of the subleading lepton is required $>15 \mathrm{GeV}$. The maximum pseudorapidity of the lepton(s) in an event should be $|\eta|<2.5$. Minimum $p_{T}$ of all jets or b-tagged jets in an event are required to be $>20 \mathrm{GeV}$ with pseudorapidity $|\eta|<2.5$. The minimum separation between a jet (or $b$-jet) and charged lepton(s) is required to be $\Delta R_{j(b) \ell}>0.4$, while the minimum separation between two charged leptons should be
$\Delta R_{\ell \ell}>0.4$, with missing transverse $E_{T}^{\text {miss }}>35 \mathrm{GeV}$. Events containing a hardronic tau lepton with $p_{T}>$ $25 \mathrm{GeV}$ are vetoed. Each event will have multiple combinations of $m_{b b}$ due to high $b$-jet multiplicity. The pairs with $m_{b b}$ closest to $M_{S}$ are assumed to be coming from decay of $S$ and passed through invariant mass cut $\left|M_{b b}-M_{S}\right|<10 \mathrm{GeV}$.

In order to illustrate the discovery potential, we consider six benchmark configurations in the minimal Majoronic model for dark radiation and dark matter [2,3] given as follows:

$$
\begin{array}{lc}
\text { Config }-1: v_{S}=1 \mathrm{TeV}, & s_{\theta}= \pm 0.1, \\
\text { Config }-2: v_{S}=1 \mathrm{TeV}, & s_{\theta}= \pm 0.3, \\
\text { Config }-3: v_{S}=10 \mathrm{TeV}, & s_{\theta}= \pm 0.1, \\
\text { Config }-4: v_{S}=10 \mathrm{TeV}, & s_{\theta}= \pm 0.3, \\
\text { Config }-5: v_{S}=100 \mathrm{TeV}, & s_{\theta}= \pm 0.1, \\
\text { Config }-6: v_{S}=100 \mathrm{TeV}, & s_{\theta}= \pm 0.3 .
\end{array}
$$

Once $v_{S}, s_{\theta}$, and $M_{S}$ are given, the invisible decay branching ratio and $B r(S \rightarrow b \bar{b})$ are fixed in this particular model. We find the result is not sensitive to the sign of $s_{\theta}$; namely, the triple and quartic scalar contributions are negligible.

The signal cross sections for different configurations after selection cuts are plotted in Fig. 1, while the background cross sections are given in the appendix for $\sqrt{s}=$ 13 and $100 \mathrm{TeV}$, respectively. To estimate signal significance, we use the following formula [33]:

$$
\mathcal{Z}=\sqrt{2\left[(S+B) \ln \left(1+\frac{S}{B}\right)-S\right]},
$$

where $S$ and $B$ are the number of signal and background events, respectively. We require $\mathcal{Z}=5$ for the $5 \sigma$ discovery and $\mathcal{Z}=3$ for $3 \sigma$ significance.

Let us have a closer look at Figs. 1 and 2. In Fig. 1, we have plotted the cross section of the signal for different values of $M_{S}$ adopting several benchmark scenarios as given in Eq. (10). The cross section contours for all the configurations increase initially and reach the maximum value at $M_{S} \sim 40 \mathrm{GeV}$, finally falling slowly towards a higher mass. The minimum $p_{T}$ cuts on the $b$-jets are too strong for $M_{S}$ below $40 \mathrm{GeV}$, causing the initial dip in the cross sections, which finally reach its maximum value $M_{S} \sim 40 \mathrm{GeV}$ and then falls again due to the drop in parton luminosity for heavier $M_{S}$. The cross section of the signal process $p p \rightarrow t \bar{t} S$ is directly proportional to $s_{\theta}^{2}$; hence, configurations with larger $\left|s_{\theta}\right|$ have larger cross sections resulting in better discovery probability. However, for fixed $s_{\theta}$ and $M_{S}$, the cross sections primarily depend on the $\mathcal{B} r(S \rightarrow b \bar{b})$. For the configurations with larger $v_{S}$, the total decay width of $S$ is 

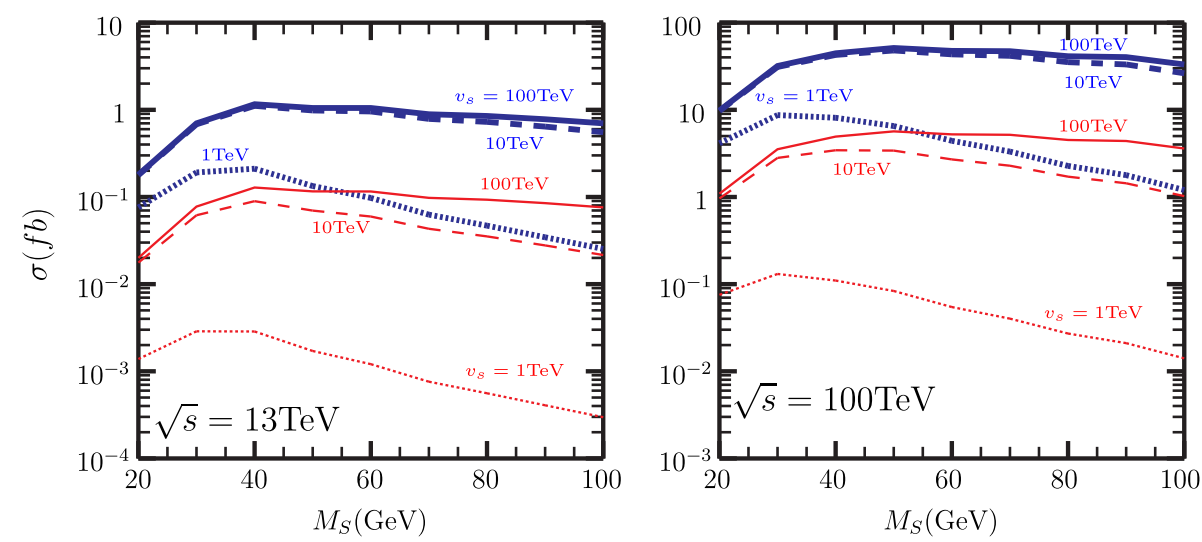

FIG. 1. Signal cross sections (fb) for different $M_{S}$ at 13 (left) and $100 \mathrm{TeV}$ (right) $p p$ collision for six different benchmark configurations as given in Eq. (10). The blue/red curves correspond to $\left|s_{\theta}\right|=0.3 / 0.1$.
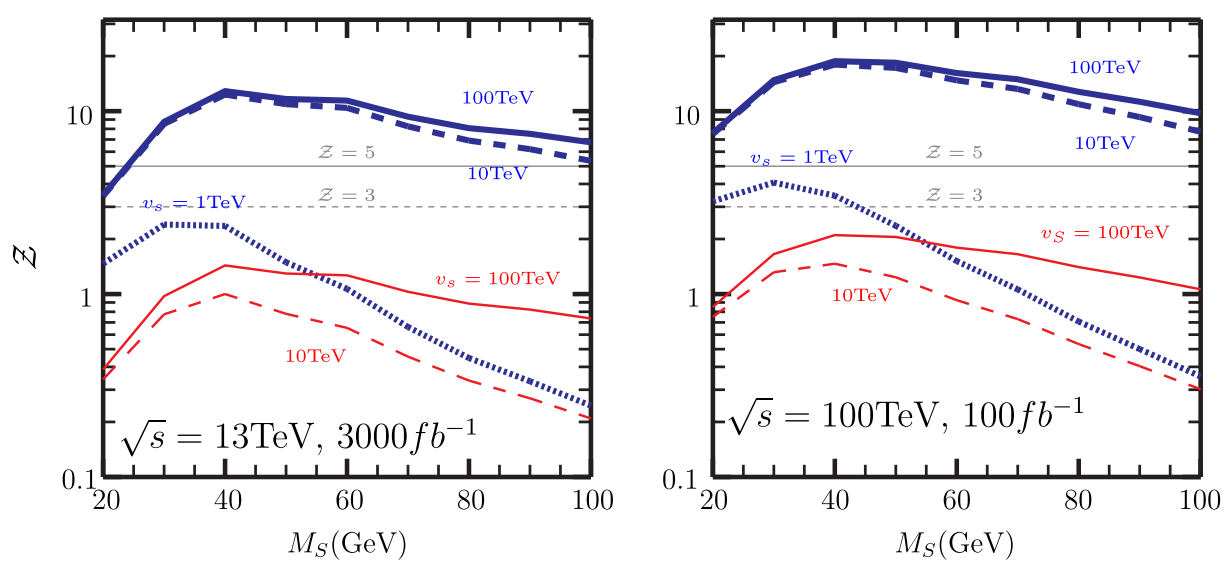

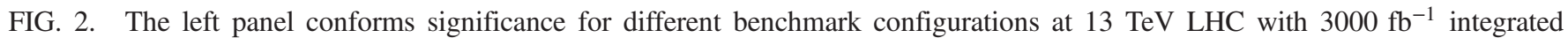
luminosity, while the right panel represents the significance for $100 \mathrm{TeV} p p$ machine with $100 \mathrm{fb}^{-1}$ integrated luminosity. Color schemes are the same as Fig. 1.

dominated by $\Gamma_{S \rightarrow b \bar{b}}{ }^{2}$ This is evident from the position of the dotted and dashed lines in Fig. 1. Furthermore, the blue solid and dashed lines almost coincide due to $\mathcal{B} r(S \rightarrow b \bar{b}) \approx 1$ for $v_{S}=10,100 \mathrm{TeV}$. However, the red solid and dashed lines are more separated than the blue ones, primarily due to $\mathcal{B} r(S \rightarrow b \bar{b})$ being lower for $v_{S}=10 \mathrm{TeV}$ than $v_{S}=100 \mathrm{TeV}$.

We restrict ourselves in the mass range $M_{S}=[20,100] \mathrm{GeV}$. For $M_{S}<20 \mathrm{GeV}$, the controlling of the background would be much more difficult due to the presence of bottomonium resonances, while with $M_{S}>100 \mathrm{GeV}$, the dominant signal process suffers interference with the SM $t \bar{t} H$ process. The strong $\left|M_{b b}-M_{S}\right|<$ $10 \mathrm{GeV}$ cut is primarily used to minimize the interference with the SM $t \bar{t} H$ process near $m_{S} \sim 100 \mathrm{GeV}$. For simplicity, we use the same invariant mass cut even for the lower $M_{S}$

\footnotetext{
${ }^{2}$ For the $M_{S}$ value under consideration, the total decay width of $S$ is nicely approximated as $\Gamma_{\text {tot }} \approx \Gamma_{S \rightarrow b \bar{b}}+\Gamma_{S \rightarrow c \bar{c}}+\Gamma_{S \rightarrow \omega \bar{\omega}}$, where $\omega$ is the singlet Majoron [2,3].
}

region. Note that we have not included QCD corrections to signal and SM background processes, which may induce some uncertainties in our results. Since our study is exploratory, it is premature to include these higher order effects. Together with all the current limits, our findings are summarized in Fig. 3. It is encouraging to see that despite the large SM background, HL-LHC13 can improve upon LEPII results on $s_{\theta}^{2}$.

We naively extrapolate our study to the $100 \mathrm{TeV}$ $p p$-collider. The cross sections for both the signal and the background are enhanced due to the rise of gluonic parton distribution at small $x$. We find the signal significance in this case with $100 \mathrm{fb}^{-1}$ integrated luminosity is slightly better than that at the HL-LHC, which may alleviate due pile-up effects. Such effects are included in a realistic experimental analysis, which we do not incorporate in our exploratory study.

\section{B. Signal at the $Z$ factory}

In the planned Z-factory option of FCC-ee, $\sim \mathcal{O}\left(10^{12}\right)$ $\mathrm{Z}$ bosons are expected each year [34]. This opens up a new 


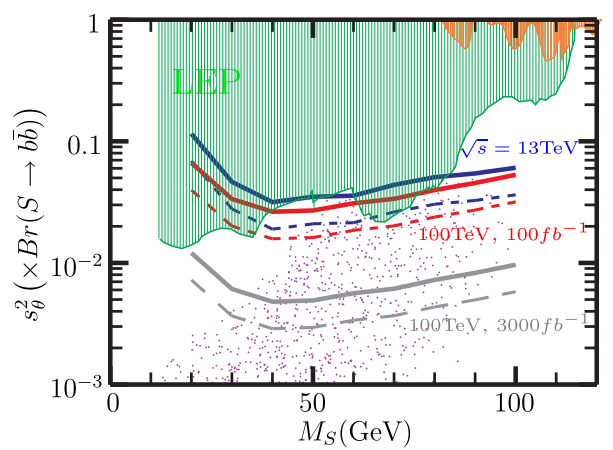

FIG. 3. $\sin ^{2} \theta(\times B r(S \rightarrow b \bar{b}))$ vs $M_{S}$. The light green area corresponds to the LEP exclusion bound [23]. The orange shade is the exclusion region from the LHC direct search $[12,13]$ which is independent of $\operatorname{Br}(S \rightarrow b \bar{b})$. The blue/red curve is for the $p p$ collider with $\sqrt{s}=13 / 100 \mathrm{TeV}$ and integrated luminosity $=$ $3000 / 100 \mathrm{fb}^{-1}$, respectively. The solid and dashed lines represent $\mathcal{Z}=5,3$ limit, respectively. The gray curves are the evidence and discovery limits at the $100 \mathrm{TeV} p p$ collider with $3000 \mathrm{fb}^{-1}$ integrated luminosity. The scattered points are the realistic configurations found in [3].

avenue to directly probe the light scalar with the rare $\mathrm{Z}$ decays. The signal will be $e^{+} e^{-} \rightarrow Z \rightarrow S f \bar{f}$, and $S$ subsequently decays into a $b \bar{b}$ pair. A useful kinetic variable $y_{b} \equiv m_{b b}^{2} / M_{Z}^{2}$ is defined where $m_{b b}$ is the invariant mass of the $b \bar{b}$ pair. The on-shell light scalar gives a very narrow resonance peak in $y_{b}$ at around $y_{b}=\left(M_{S} / M_{Z}\right)^{2}$ and stands out from the continuous SM background. The signal branching ratio is given by [35]

$B r(Z \rightarrow S f \bar{f})=s_{\theta}^{2} \times F\left(M_{S} / M_{Z}\right) \times B r(Z \rightarrow f \bar{f})$,

where

$$
\begin{aligned}
F(r)= & \frac{G_{F} M_{W}^{2}}{24 \sqrt{2} \pi^{2} c_{W}^{2}}\left[\frac{3 r\left(r^{4}-8 r^{2}+20\right)}{\sqrt{4-r^{2}}} \cos ^{-1}\left(\frac{r}{2}\left(3-r^{2}\right)\right)\right. \\
& \left.-3\left(r^{4}-6 r^{2}+4\right) \ln r-\frac{1}{2}\left(1-r^{2}\right)\left(2 r^{4}-13 r^{2}+47\right)\right] .
\end{aligned}
$$

Here, $c_{W}\left(s_{W}\right)$ is the shorthand for the weak mixing $\cos \theta_{W}\left(\sin \theta_{W}\right)$. One of the signals we are interested in is $Z \rightarrow \nu \bar{\nu} S ; S \rightarrow b \bar{b}$, and the SM background is the 4-body decay $Z \rightarrow b \bar{b} \nu \bar{\nu}$. To quantify the discovery potential at the future $Z$-factory, we calculate the significant $\mathcal{Z}$. The signal will be

$$
S=s_{\theta}^{2} \times F\left(r_{S}\right) \times B r(Z \rightarrow \nu \bar{\nu}) \times B r(S \rightarrow b \bar{b}) \times N_{Z},
$$

where $r_{S}=\left(M_{S} / M_{Z}\right), N_{Z}$ is the fiducial number of Z-bosons, and we sum over all neutrino species. We use CalcHep [36] to calculate numerically the SM differential decay width, $d \Gamma^{\mathrm{SM}}(Z \rightarrow b \bar{b} \nu \bar{\nu}) / d y_{b}$ (see Fig. 10 in [3]). Then, the signal background is obtained by integration over the continuous distribution in the vicinity of $y_{b}=r_{S}^{2}$

$$
\mathrm{B}=N_{Z} \times \int_{r_{S}^{2}-\delta y_{b}}^{r_{S}^{2}+\delta y_{b}} d y_{b} \frac{d \Gamma^{\mathrm{SM}}(Z \rightarrow b \bar{b} \nu \bar{\nu})}{d y_{b}} .
$$

Assuming the invariant mass resolution at the FCC-ee is $1 \mathrm{GeV}$, then $\delta y_{b}$ is roughly $2 \frac{M_{s} \times 1 \mathrm{GeV}}{M_{Z}^{2}} \sim \frac{r_{S}}{45}$. Taking $N_{Z}=$ $10^{12}$ and $\operatorname{Br}(Z \rightarrow \nu \bar{\nu})=0.2000(6)$ [37], the curves of $\mathcal{Z}=3,5$ are displayed in Fig. 5. Not surprisingly, at a Z-factory, one could probe the new light scalar in the mass range from 20 to $80 \mathrm{GeV}$ with mixing orders of magnitude smaller than the current LEP bound and far better than a high energy pp collider.

\section{Signal at the Higgs factory}

The plan for a Higgs factory is an $e^{+} e^{-}$collider operating at $\sqrt{s}=240-250 \mathrm{GeV}$ and luminosity of a few $\mathrm{ab}^{-1}$. For definiteness, we take $\sqrt{s}=240 \mathrm{GeV}$ and, to take into account the detection efficiency, use an integrated fiducial luminosity $\mathfrak{L}=1 \mathrm{ab}^{-1}$ as the benchmark. The tree-level scattering cross section is

$\sigma\left(e^{+} e^{-} \rightarrow Z S\right)=\frac{s_{\theta}^{2} \pi \alpha^{2}}{3 s_{W}^{4} c_{W}^{4}}\left(g_{e L}^{2}+g_{e R}^{2}\right) \frac{p_{c m}\left(p_{c m}^{2}+3 M_{Z}^{2}\right)}{\sqrt{s}\left(s-M_{Z}^{2}\right)^{2}}$,

where $\quad g_{e L}=s_{W}^{2}-\frac{1}{2}, \quad g_{e R}=s_{W}^{2}, \quad$ and $\quad p_{c m}=$ $\sqrt{\left(s+M_{S}^{2}-M_{Z}^{2}\right)^{2} /(4 s)-M_{S}^{2}}$ is the CM momentum of the $S$. To be more specific, we consider the final states $Z \rightarrow \mu \bar{\mu}$ and $S \rightarrow b \bar{b}$ as an example. Then, the number of signal events will be

$S=\mathfrak{Q} \times \sigma\left(e^{+} e^{-} \rightarrow Z S\right) \times B r(S \rightarrow b \bar{b}) \times B r(Z \rightarrow \mu \bar{\mu})$

and $\operatorname{Br}(Z \rightarrow \mu \bar{\mu})=3.366(7) \%$ [37]. The SM background is dominated by the t-channel $e^{+} e^{-} \rightarrow Z+Z$ diagram, and the complete tree-level contribution is evaluated numerically by CalcHEP. We applied two cuts, (1) $\left|M_{\mu \bar{\mu}}-M_{Z}\right|<1 \mathrm{GeV}$ and (2) $\left|E_{\mu \bar{\mu}}-\sqrt{M_{Z}^{2}+p_{c m}^{2}}\right|<1 \mathrm{GeV}$, to suppress the SM background. See Fig. 4 for the result. One can clearly see the huge peak around $M_{Z}$ due to the $Z$ resonance, and the photon contribution accounts for the rise at the low energy end.

Due to the finite energy resolution (for that we use $\delta m_{b \bar{b}}=1 \mathrm{GeV}$ as the benchmark), the number of SM background events is given by

$$
B=\mathfrak{Q} \times \int_{m_{b \bar{b}}-\delta m}^{m_{b \bar{b}}+\delta m} \frac{\sigma_{\mathrm{SM}}(Z \rightarrow \mu \bar{\mu} b \bar{b})}{d m_{b \bar{b}}} .
$$

The curves for signal significance $\mathcal{Z}=3,5$ are shown in Fig. 5 along with other results depicted in Fig. 3. It can be seen that the sensitivity to $s_{\theta}^{2} \cdot B r$ is poorer when $M_{S}$ is around the Z-pole due to the large intrinsic SM 


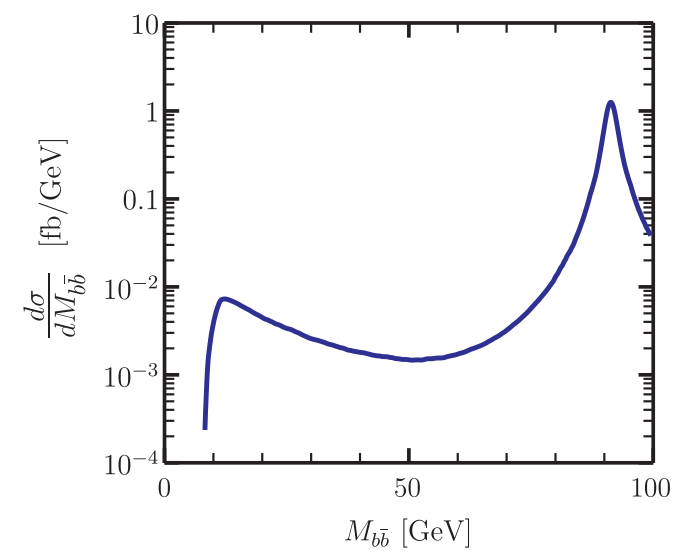

FIG. 4. SM background for $e^{+} e^{-} \rightarrow Z S$ at the Higgs factory with $\sqrt{s}=240 \mathrm{GeV}$. See the text for details.

background. However, the shear statistics make the sensitivity roughly two orders of magnitude better than that at the LEP.

\section{Muon $(g-2)$}

The current status of the muon anomalous magnetic moment is $a_{\mu}^{\exp }-a_{\mu}^{\mathrm{SM}}=2.88(80) \times 10^{-9}$ [37]. Both the modified SM Higgs coupling and the presence of the light scalar contribute to $\triangle a_{\mu}$

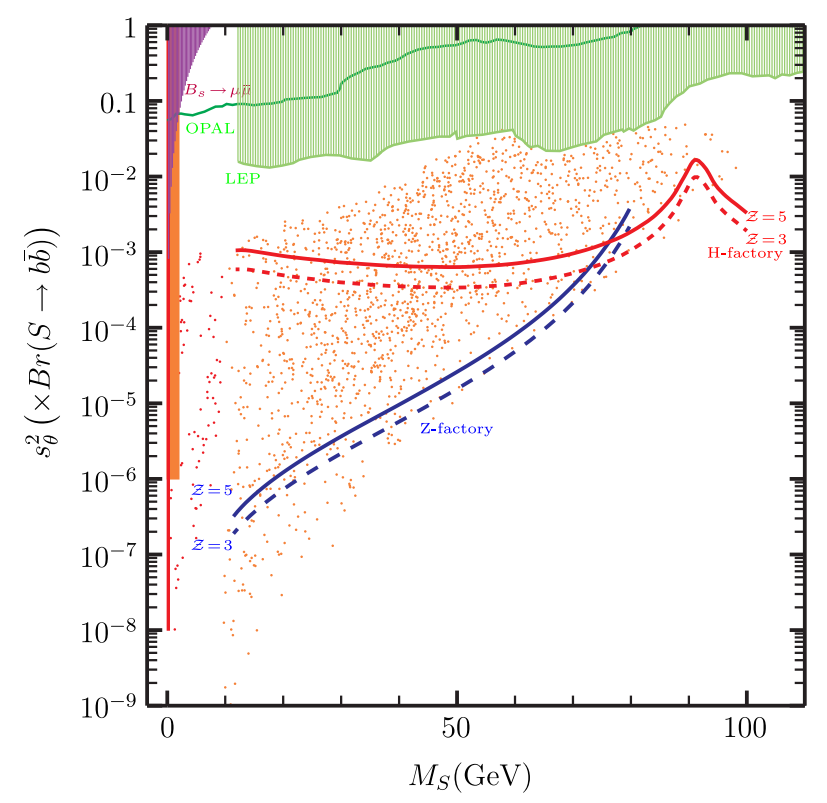

FIG. 5. $\sin ^{2} \theta(\times B r(S \rightarrow b \bar{b}))$ vs $M_{S}$. The left most two are from rare $\mathrm{B}$ and $\mathrm{K}$ decays. The upper-left region in purple is the two sigma exclusion from the $B_{s} \rightarrow \mu \bar{\mu}$. The light green area corresponds to the LEP exclusion bound [23]. The dark green curve is the decay mode independent bound from OPAL[38]. The scattered points are the realistic configurations found in [3]. The blue(red) dashed/solid curve is for signal significant $\mathcal{Z}=3,5$ at the $\mathrm{Z}$ (Higgs) factory, respectively.

$$
\triangle a_{\mu}=s_{\theta}^{2} \frac{G_{F} m_{\mu}^{2}}{4 \pi^{2} \sqrt{2}}\left[G\left(M_{S}^{2} / m_{\mu}^{2}\right)-G\left(M_{H}^{2} / m_{\mu}^{2}\right)\right]
$$

at a 1-loop level, where

$$
G(x)=\int_{0}^{1} d y \frac{y^{2}(2-y)}{y^{2}+x(1-y)} .
$$

Note that $G(0)=3 / 2$; therefore, $\triangle a_{\mu} \simeq 3.49 \times s_{\theta}^{2} \times 10^{-9}$ when $M_{S} \ll m_{\mu}$. The modification moves up the SM prediction and alleviates the tension between theory and experiments. More precisely, to explain the discrepancy at the two sigma lower bound, the mixing has to be

$$
s_{\theta}^{2}=\frac{0.5447}{G\left(M_{S}^{2} / m_{\mu}^{2}\right)-9.171 \times 10^{-6}} .
$$

The above equation has a solution only if $M_{S}<0.1 \mathrm{GeV}$ and $s_{\theta}^{2} \gtrsim 0.5$ which has been ruled out by the $K^{+} \rightarrow \pi^{+}+$nothing. Although the light singlet scalar provides a positive contribution to $a_{\mu}$, it alone cannot accommodate the current discrepancy between theory and experiment.

$$
\text { E. } B_{s} \rightarrow \mu \bar{\mu}
$$

The presence of the light scalar also contributes to the rare process $B_{s} \rightarrow \mu \bar{\mu}$. This transition is governed by the effective Hamiltonian

$\mathcal{H}_{\mathrm{eff}}=-\frac{G_{F}}{\sqrt{2}} \frac{\alpha}{\pi s_{W}^{2}} V_{t b} V_{t S}^{*}\left(C_{10} \hat{O}_{10}+C_{S} \hat{O}_{S}+C_{P} \hat{O}_{P}\right)$

where

$$
\begin{aligned}
\hat{O}_{10} & =\left(\bar{s} \gamma_{\mu} \hat{L} b\right)\left(\bar{\mu} \gamma^{\mu} \gamma_{5} \mu\right), \\
\hat{O}_{S} & =\frac{m_{\mu} m_{b}}{M_{W}^{2}}(\bar{s} \hat{R} b)(\bar{\mu} \mu), \\
\hat{O}_{P} & =\frac{m_{\mu} m_{b}}{M_{W}^{2}}(\bar{s} \hat{R} b)\left(\bar{\mu} \gamma_{5} \mu\right),
\end{aligned}
$$

where $\hat{R}=\frac{1+\gamma_{5}}{2}$ and $\hat{L}=\frac{1-\gamma_{5}}{2}$. Note that the vector part of the lepton current in $\hat{O}_{10}$ does not contribute in this process when contracted with the meson momentum. In SM, the Z-penguin or $\hat{O}_{10}$ dominates the decay. The complete 1-loop expressions of the Wilson coefficients, $C_{S}$ and $C_{P}$, can be found in [39], and we do not repeat that here. The key is that the SM Higgs gives a negative contribution $-3 m_{t} / 8 M_{H}$ to $C_{S}^{\mathrm{SM}}(\simeq-0.939)$. The branching ratio is given by

$$
\begin{aligned}
\operatorname{Br}\left(B_{s} \rightarrow \mu \bar{\mu}\right)= & \frac{\tau_{B_{s}} G_{F}^{4} M_{W}^{4}}{8 \pi^{5}}\left|V_{t b} V_{t s}^{*}\right|^{2} f_{B_{s}}^{2} M_{B_{S}} m_{\mu}^{2} \\
& \times \sqrt{1-4 m_{\mu}^{2} / M_{B_{s}}^{2}}\left(|\mathbf{P}|^{2}+|\mathbf{S}|^{2}\right),
\end{aligned}
$$


where

$$
\begin{aligned}
& \mathbf{P}=C_{10}+\frac{M_{B_{s}}^{2}}{2 M_{W}^{2}} \frac{m_{b}}{m_{b}+m_{s}} C_{P}, \\
& \mathbf{S}=\sqrt{1-4 m_{\mu}^{2} / M_{B_{s}}^{2}} \frac{M_{B_{s}}^{2}}{2 M_{W}^{2}} \frac{m_{b}}{m_{b}+m_{s}} C_{S} .
\end{aligned}
$$

The reduction of the SM Higgs coupling and the light scalar modify the scalar penguin contribution to this decay, and

$$
C_{S}^{\mathrm{SM}} \Rightarrow C_{S}^{\mathrm{SM}}-\frac{3}{8} s_{\theta}^{2}\left(\frac{m_{t}^{2}}{M_{S}^{2}}-\frac{m_{t}^{2}}{M_{H}^{2}}\right)
$$

When $M_{S}<M_{H},\left|C_{S}\right|>\left|C_{S}^{\mathrm{SM}}\right|$. On the other hand, $C_{10}$ and $C_{P}$ remain the same. Therefore, the $B_{s} \rightarrow \mu \bar{\mu}$ branching ratio is larger than the SM one in the general Higgs portal model with a light singlet scalar. What is experimentally measured is the time averaged decay branching ration $\overline{\operatorname{Br}}\left(B_{s} \rightarrow \mu \bar{\mu}\right)$ due to the sizable $B_{s}-\overline{B_{s}}$ oscillation [40]. The current experimental result agrees with the SM prediction [41]

$$
\bar{R}=\frac{\overline{\operatorname{Br}}\left(B_{s} \rightarrow \mu \bar{\mu}\right)_{\exp }}{\overline{\operatorname{Br}}\left(B_{s} \rightarrow \mu \bar{\mu}\right)_{\mathrm{SM}}}=0.84 \pm 0.16,
$$

where the results measured at LHCb [42] and CMS [43] and the SM prediction from [44] are used.

Numerically, at two sigma $(\bar{R}<1.16)$, we find

$$
s_{\theta}^{2}<\frac{496.06}{m_{t}^{2} / M_{S}^{2}-1.91}
$$

for $M_{S}<7.76 \mathrm{GeV}$. That the mixing can be constrained is understandable since the negative scalar penguin contribution becomes important and increases the branching ratio when the second scalar is light. However, the constraint on $s_{\theta}^{2}$ from $B_{s} \rightarrow \mu \bar{\mu}$ for $M_{S}<7.76 \mathrm{GeV}$ cannot compete with the limit given by the decay-mode independent searches at LEP [38].

\section{SUMMARY}

We have analyzed the mixing of a singlet scalar with the SM Higgs boson in the challenging mass range from 10 to $100 \mathrm{GeV}$. We found that by using the signal of $4 b$-jets $+\geq$ $1 \ell+$ MET one can successfully probe this mass range with limits on the mixing angle $\sim$ an order better than the current LEPII results. We have used very conservative cuts and search criteria established by ATLAS and CMS Collaborations. We did not use more advanced techniques such as boosted $t$-quarks nor neural networks which will improve our exploratory study. The significance of our results only applies to HL-LHC due to the smallness of the cross section for $p p \rightarrow t \bar{t} S$. An order of magnitude improvement over the LEPII results is achievable at LHC13 (see Fig. 3) for $80 \mathrm{GeV}<M_{S}<110 \mathrm{GeV}$. This analysis is then naively extended to a future $100 \mathrm{TeV} \mathrm{pp}$ collider. Our calculation shows that not much is gained if the luminosity is relatively low. In contrast, a high luminosity $100 \mathrm{TeV}$ collider can improve on the LEPII limit by up to two orders of magnitude in a mixing angle scan. We realize that such a futuristic machine will have different systematics and experimental challenges and theoretical uncertainties that make such an extrapolation speculative at best. Nonetheless, we find the result intriguing.

The discovery potential of the $125 \mathrm{GeV}$ Higgs in the $t \bar{t} H$ production with $H \rightarrow b \bar{b}$ decay has been extensively discussed in Refs. [15,45-47]. With the collection of $3 \mathrm{ab}^{-1}$ data, the $\bar{t} H$ signal strength can be probed up to $\sim 20 \%$ level of the SM prediction [47]. If measured, a lower deviation of the $t \bar{t} H$ signal strength from the SM prediction can be attributed to the mixing between the singlet scalar $S$ and $125 \mathrm{GeV} H$. However, the mass of $S$ cannot be ascertained from this alone. Thus, such a deviation will act as an indirect probe for the singlet $S$ in our model. Clearly, a direct probe via $t \bar{t} S, S \rightarrow b \bar{b}$ would be essential. We also remark that a search for light $C P$-even resonance in association with $t \bar{t}$ production, based on a simplified model, has been discussed in [48].

The signal for light singlet scalar discovery we studied hinges on a sizable $b \bar{b}$ decay. For models which have large portal Higgs to dark sector coupling (HD), our results can be scaled by scaling the $b \bar{b}$ branching ratio. If the HD is large, then an efficient missing energy signal will have to be used. However, we have not found a signal in which $S \rightarrow$ invisible can be detected above the SM background at the LHC for this range of $M_{S}$.

As a comparison, we also studied how the Z-factory and Higgs factory options for a future $e^{+} e^{-}$collider can be used to study the light scalars. We found that the $Z \rightarrow S \nu \bar{\nu}$; $S \rightarrow b \bar{b}$ is the most promising signal to search for. If not found, the limit set on the mixing angle will be 3-4 orders of magnitude better than LEPII. We also note that due to the stringent bound from $K \rightarrow \pi \nu \nu$, the muon g-2 discrepancy cannot be explained alone by the presence of a very light scalar. Moreover, the recent $B_{s} \rightarrow \mu \bar{\mu}$ data does not provide a better constraint on $s_{\theta}^{2}$ for $2<M_{S}<7.7 \mathrm{GeV}$ than that from the branching ratio independent light scalar search at LEP [38].

\section{ACKNOWLEDGMENTS}

W. F. C. is supported by the Taiwan Ministry of Science and Technology under Grants No. 106-2112-M-007-009MY3 and No. 105-2112-M-007-029. T. M. is supported by the Ministry of Science and Technology of R. O.C. under Grant No. MOST-106-2811-M-002-187. T. M. thanks Stathes Paganis for discussions. 


\section{APPENDIX: BACKGROUND CROSS SECTIONS}

The cross sections of the different background processes of the $(4 j, 4 b, 1 \ell)$ process for different $M_{S}$, with $\sqrt{s}=13$ and $100 \mathrm{TeV} p p$ collision are presented in Tables I and II, respectively.

TABLE I. Cross section in $\mathrm{fb}$ of different background processes for after applying selection cuts. The cross section is estimated at $\sqrt{s}=13$ TeV LHC.

\begin{tabular}{lcccccccr}
\hline \hline $\begin{array}{l}M_{S} \\
(\mathrm{GeV})\end{array}$ & $\begin{array}{c}t \bar{t}+\text { h.f. } \\
\text { jets }\end{array}$ & $\begin{array}{c}t \bar{t}+\text { l.f. } \\
\text { jets }\end{array}$ & $\begin{array}{c}\text { Single } \\
\text { top }\end{array}$ & $t \bar{t} Z$ & $t \bar{t} H$ & $t W H$ & Others & $\begin{array}{c}\text { Total } \\
\text { BG }\end{array}$ \\
\hline 20 & 7.75 & 0.116 & 0.005 & 0.009 & 0.035 & 0.003 & 0.005 & 7.923 \\
30 & 18.41 & 0.321 & 0.013 & 0.03 & 0.148 & 0.009 & 0.032 & 18.936 \\
40 & 23.26 & 0.486 & 0.018 & 0.074 & 0.279 & 0.013 & 0.042 & 24.172 \\
50 & 22.29 & 0.605 & 0.02 & 0.126 & 0.418 & 0.019 & 0.045 & 23.523 \\
60 & 23.26 & 0.706 & 0.023 & 0.200 & 0.501 & 0.022 & 0.059 & 24.771 \\
70 & 25.20 & 0.839 & 0.024 & 0.266 & 0.590 & 0.024 & 0.076 & 27.019 \\
80 & 31.01 & 0.911 & 0.026 & 0.316 & 0.665 & 0.029 & 0.081 & 33.038 \\
90 & 30.04 & 0.912 & 0.026 & 0.326 & 0.711 & 0.032 & 0.085 & 32.132 \\
100 & 30.04 & 0.922 & 0.025 & 0.292 & 0.787 & 0.036 & 0.081 & 32.183 \\
\hline \hline
\end{tabular}

TABLE II. Same as Table I but for $\sqrt{s}=100 \mathrm{TeV}$ LHC.

\begin{tabular}{lcccccccc}
\hline \hline $\begin{array}{l}M_{S} \\
(\mathrm{GeV})\end{array}$ & $\begin{array}{c}t \bar{t}+\text { h.f. } \\
\text { jets }\end{array}$ & $\begin{array}{c}t \bar{t}+\text { l.f. } \\
\text { jets }\end{array}$ & $\begin{array}{c}\text { Single } \\
\text { top }\end{array}$ & $t \bar{t} Z$ & $t \bar{t} H$ & $t W H$ & Others & $\begin{array}{c}\text { Total } \\
\text { BG }\end{array}$ \\
\hline 20 & 156.02 & 4.90 & 0.10 & 0.48 & 2.20 & 0.17 & 0.1 & 163.97 \\
30 & 429.04 & 13.47 & 0.26 & 1.57 & 8.36 & 0.50 & 0.69 & 453.89 \\
40 & 507.05 & 20.36 & 0.38 & 3.98 & 15.33 & 0.79 & 1.29 & 549.18 \\
50 & 702.07 & 24.96 & 0.43 & 7.27 & 21.47 & 1.05 & 1.39 & 758.64 \\
60 & 780.08 & 28.89 & 0.46 & 9.28 & 26.72 & 1.31 & 1.29 & 848.03 \\
70 & 897.08 & 33.83 & 0.49 & 13.51 & 30.45 & 1.52 & 1.57 & 978.45 \\
80 & 936.09 & 36.29 & 0.51 & 17.97 & 35.15 & 1.67 & 2.48 & 1030.16 \\
90 & 1170.11 & 35.75 & 0.51 & 16.64 & 39.42 & 1.97 & 1.98 & 1266.38 \\
100 & 1053.10 & 35.18 & 0.51 & 13.75 & 42.95 & 2.23 & 1.78 & 1149.50 \\
\hline \hline
\end{tabular}

[1] W. F. Chang, J. N. Ng, and J. M. S. Wu, Phys. Lett. B 730, 347 (2014).

[2] W. F. Chang and J. N. Ng, Phys. Rev. D 90, 065034 (2014).

[3] W. F. Chang and J. N. Ng, J. Cosmol. Astropart. Phys. 07 (2016) 027.

[4] F. S. Queiroz and K. Sinha, Phys. Lett. B 735, 69 (2014).

[5] S. Profumo, M. J. Ramsey-Musolf, and G. Shaughnessy, J. High Energy Phys. 08 (2007) 010.

[6] M. Gonderinger, H. Lim, and M. J. Ramsey-Musolf, Phys. Rev. D 86, 043511 (2012).

[7] J. N. Ng and A. de la Puente, Eur. Phys. J. C 76, 122 (2016).

[8] B. Patt and F. Wilczek, arXiv:hep-ph/065188.

[9] W. F. Chang, J. N. Ng, and J. M. S. Wu, Phys. Rev. 74, 095005 (2006).
[10] ATLAS and CMS Collaborations, Report No. ATLASCONF-2015-044.

[11] S. Dawson et al., arXiv:1310.8361.

[12] T. Robens and T. Stefaniak, Eur. Phys. J. C 75, 104 (2015).

[13] G. Dupuis, J. High Energy Phys. 07 (2016) 008.

[14] J. D. Wells, In Perspectives on LHC Physics, edited by G. Kane and A. Pierce (World Scientific Publishing Company, Singapore, 2008).

[15] T. Plehn, G. P. Salam, and M. Spannowsky, Phys. Rev. Lett. 104, 111801 (2010).

[16] S. Bock, R. Lafaye, T. Plehn, M. Rauch, D. Zerwas, and P. M. Zerwas, Phys. Lett. B 694, 44 (2010).

[17] C. Englert, T. Plehn, D. Zerwas, and P. M. Zerwas, Phys. Lett. B 703, 298 (2011). 
[18] C. Englert, T. Plehn, M. Rauch, D. Zerwas, and P. M. Zerwas, Phys. Lett. B 707, 512 (2012).

[19] V. Martin Lozano, J. M. Moreno, and C. B. Park, J. High Energy Phys. 08 (2015) 004.

[20] A. Falkowski, C. Gross, and O. Lebedev, J. High Energy Phys. 05 (2015) 057.

[21] D. Lopez-Val and T. Robens, Phys. Rev. D 90, 114018 (2014).

[22] G. Ria and D. Meloni, arXiv:1705.11126.

[23] R. Barate et al. (ALEPH and DELPHI and L3 and OPAL Collaborations and LEP Working Group for Higgs boson searches), Phys. Lett. B 565, 61 (2003).

[24] L. A. Anchordoqui, P. B. Denton, H. Goldberg, T. C. Paul, L. H. M. Da Silva, B. J. Vlcek, and T. J. Weiler, Phys. Rev. D 89, 083513 (2014).

[25] ATLAS Collaboration, Report No. ATLAS-CONF-2016080.

[26] J. Alwall, R. Frederix, S. Frixione, V. Hirschi, F. Maltoni, O. Mattelaer, H.-S. Shao, T. Stelzer, P. Torrielli, and M. Zaro, J. High Energy Phys. 07 (2014) 079.

[27] R. D. Ball, V. Bertone, S. Carrazza, L. Del Debbio, S. Forte, A. Guffanti, N. P. Hartland, and J. Rojo (NNPDF Collaboration), Nucl. Phys. B877, 290 (2013).

[28] T. Sjostrand, S. Mrenna, and P. Z. Skands, J. High Energy Phys. 05 (2006) 026.

[29] J. Alwall et al., Eur. Phys. J. C 53, 473 (2008).

[30] J. de Favereau, C. Delaere, P. Demin, A. Giammanco, V. Lemaître, A. Mertens, and M. Selvaggi (DELPHES 3 Collaboration), J. High Energy Phys. 02 (2014) 057.

[31] ATLAS Collaboration, Report No. ATLAS-CONF-2014058.

[32] A. Alloul, N. D. Christensen, C. Degrande, C. Duhr, and B. Fuks, Comput. Phys. Commun. 185, 2250 (2014).
[33] G. Cowan, K. Cranmer, E. Gross, and O. Vitells, Eur. Phys. J. C 71, 1554 (2011); 73, 2501(E) (2013).

[34] M. Bicer et al. (TLEP Design Study Working Group), J. High Energy Phys. 01 (2014) 164 (2014).

[35] J. F. Gunion, H. E. Haber, G. L. Kane, and S. Dawson, Front. Phys. 80, 1 (2000).

[36] A. Belyaev, N. D. Christensen, and A. Pukhov, Comput. Phys. Commun. 184, 1729 (2013).

[37] C. Patrignani et al. (Particle Data Group), Chin. Phys. C 40, 100001 (2016).

[38] G. Abbiendi et al. (OPAL Collaboration), Eur. Phys. J. C 27, 311 (2003).

[39] X. Q. Li, J. Lu, and A. Pich, J. High Energy Phys. 06 (2014) 022 .

[40] K. De Bruyn, R. Fleischer, R. Knegjens, P. Koppenburg, M. Merk, A. Pellegrino, and N. Tuning, Phys. Rev. Lett. 109, 041801 (2012).

[41] R. Fleischer, D. G. Espinosa, R. Jaarsma, and G. TetlalmatziXolocotzi, Eur. Phys. J. C 78, 1 (2018).

[42] R. Aaij et al. (LHCb Collaboration), Phys. Rev. Lett. 118, 191801 (2017).

[43] S. Chatrchyan et al. (CMS Collaboration), Phys. Rev. Lett. 111, 101804 (2013).

[44] R. Fleischer, R. Jaarsma, and G. Tetlalmatzi-Xolocotzi, J. High Energy Phys. 05 (2017) 156.

[45] M. R. Buckley and D. Goncalves, Phys. Rev. Lett. 116, 091801 (2016).

[46] H. L. Li, P. C. Lu, Z. G. Si, and Y. Wang, Chin. Phys. C 40, 063102 (2016).

[47] N. Moretti, P. Petrov, S. Pozzorini, and M. Spannowsky, Phys. Rev. D 93, 014019 (2016).

[48] M. J. Dolan, M. Spannowsky, Q. Wang, and Z. H. Yu, Phys. Rev. D 94, 015025 (2016). 